\title{
Are Internet Tunnels Worthwhile?
}

\author{
Habiba Akter \\ School of Electronic Engineering and Computer Science \\ Queen Mary, University of London \\ London, United Kingdom \\ h.akter@qmul.ac.uk
}

\begin{abstract}
Despite a significant increase in capacity of the Internet regional congestion remains an issue at certain times of day. Dimensioning the system to provide minimal delay under these transient conditions would be uneconomical, particularly as various forms of application data are more or less sensitive to these delays, as are different end-users. We therefore investigate a scheme that allows end-users to selectively exploit a sequence of mini tunnels along a path from their origin to a chosen destination. We assume the availability of such tunnels is advertised centrally through a broker, with the cooperation of the Autonomous System (AS) domain operators, allowing end-users to use them if so desired. The closest analogy this scheme is that of a driver choosing to use one or more toll roads along a route to avoid potential congestion or less desirable geographic locations. It thus takes the form of a type of loose source routing. Furthermore, the approach avoids the need for inter-operator cooperation, although such cooperation provides a means of extending tunnels across AS peers. In this paper we ascertain the benefit in terms of delay for a given degree of tunnel presence within a portion of the Internet. The expectation is that a relatively small number of tunnels may be sufficient to provide worthwhile improvements in performance for some users at least.
\end{abstract}

Keywords-Internet, loose source routing, tunnelling, traffic congestion.

\section{INTRODUCTION}

\section{A. Motivation for Tunnels}

Although the Internet is both robust and flexible due to its federated nature, providing delivery of time-critical data that traverses multiple Autonomous System (AS) domains is still challenging. This is hampered by the unwillingness of network operators to support inter-operator signalling coupled with the control of the associated forwarding infrastructure [1]. Mechanisms for such signalling exist with functional entities such as the ITU Resource and Admission Control Function (RACF) and the IETF Path Computation Element (PCE) [2]. Although these operator-owned control plane entities have been proposed and refined over many years, their adoption outside of the academic community is no closer.

The signalling mechanism to support reliable end-to-end delivery either remains limited to exploiting AS path information provided by the Border Gateway Protocol (BGP) or the construction of overlay networks. Despite much published material on the RACF and PCE concerning how these entities could function across multiple inter-provider domains $[3,4,5]$, practical schemes are no nearer adoption.

However, the focus of this paper is not concerned with establishing end-to-end paths, or tunnels spanning multiple AS domains. Rather we aim to design a scheme where the tunnels available in the network will be advertised by a

\author{
Chris Phillips \\ School of Electronic Engineering and Computer Science \\ Queen Mary, University of London \\ London, United Kingdom \\ chris.i.phillips@qmul.ac.uk
}

\begin{abstract}
"Service Broker" to the end-users, giving the users (i.e. typically via some automated path selection algorithm) the opportunity to select which of those to use, if any, while sending their data from a source to destination address. We assume that at least some operators will be cooperative, letting the broker know about the available tunnels and their characteristics, since we consider a mechanism of financial recompense is in place. Furthermore we envision an entity being present at the users' access point, which would select the most "appropriate" path for a given data stream depending on constraints such as the amount of money the user is ready to pay, the end-to-delay, and the flow content for example.
\end{abstract}

The motivation for tunnelling over segments or the entire end-to-end path across the Internet is to overcome limitations inherent in the traditional next-hop forward ing mechanism. With next-hop forwarding the path taken by the traffic is determined by the router node at each "hop" point using information held in its Forwarding Information Base (FIB). The FIB data is typically constructed based on automatically configured routing information obtained via intra- and intergateway routing protocols along with operator policy filtering [6]. This presents two key issues. First, the end-user has no say in how their data is forwarded. Second, lack of traffic differentiation means that information flows along paths based on a simple "least cost" metric leading to load imbalances and "best effort" equal treatment of all traffic irrespective of its importance to the user.

A tunnelling mechanism, e.g., a classification and label switching mechanism can be used to address both of these issues. Tunnelling has already been implemented using various technologies [7]. We aim to give end-users some control over choosing the path their data flows by making the presence of these tunnels visible, advertised centrally using a broker, along with a means of steering traffic in sequence between them. Although the broker we are proposing is expected to know where the tunnels are, along with the characteristics, it does not need to know how the tunnels are established or operated. Operator security is not compromise as the details of the technology used to provide the tunnels is hidden, and their establishment and maintenance remains fully under the control of the operator.

Users can choose to use the tunnels, if they wish, for a nominal fee. The idea of charging customers for better service is not new [8]. However, in our case electing to use the tunnels is optional and it is up to the user which flow(s) are directed through them. As such, some customers may be happy to pay to obtain flow transport with a better Quality of Experience (QoE).

In our proposal, the end-user will be the one to decide whether specific tunnels will be used or not, knowing the "financial cost" and the expected benefits. Operators are expected to cooperate as they receive extra revenue by 
providing the tunnels. However, these tunnels, at least initially, only straddle ingress to egress points of specific AS domains between AS Border Routers (ASBRs). The location, delay, cost and perhaps resilience of these tunnels (comprising an IP address of the ingress ASBR and additional information) are passed to the broker. An entity at the end-user's access point can see the information advertised by the broker and optionally decide to direct traffic flows via one or more tunnels if the perceived benefits are sufficient relative to the cost involved.

\section{B. Net Neutrality}

The term "net neutrality" was first used in 2003, by Tim $\mathrm{Wu}$, as an augmentation of the idea of "common carrier" (which transports data for any person or company with taking the responsibility of any possible loss) for telephone systems [9]. Net neutrality is the idea stated as "all Internet traffic should be treated equally" [10]. According to this idea, Internet Service Providers (ISPs) and the governments regulating the Internet treat all the data equally, without making any discrimination or taking different charges by user, content, website, platform, application, type of attached documents, e.g., emails, audio, video, or mode of communication [11]. Hence, according to this policy, the ISPs cannot have the capability of prioritising any data over the others while sending it from the source to the expected destination.

Thinking generally, it can be easily stated that a few milliseconds' delay while sending an email will not bother the sender or receiver much. On the other hand, the same amount of delay in a video streaming will leave negative impact on the Quality of Experience (QoE) of the user. Indeed, the argument against net neutrality is supported by economists, ISPs, and technologists. Even operators like Comcast, AT\&T, Verizon, and Alcatel-Lucent, oppose net neutrality.

A motivation behind our research is that from the point of view of an end-user, treating all the traffic in the Internet equally creates problems. There is much debate concerning the topic of whether it is a good idea to give operators the chance to decide how different traffic should be treated. However our vision is not about charging users for the services, rather it gives the users the opportunity to choose if they want to pay for getting a better service and also provides some control over how their traffic moves across the Internet. Hence, in a way, we are not against the net neutrality, rather we aim to give more control to the users to decide how they want their traffic to be handled by the Internet.

\section{EXAMPLE ARCHITECTURE}

\section{A. The Tunnelling Framework}

The basic architecture of the AS-Domain tunnelling framework is shown in Fig. 1. The tunnels shown are assumed to have been setup and maintained by the specific network operators using whatever means they wish. This could involve the use of PCE/RACF signalling; however, this is not essential. The presence of the tunnels is advertised via the Directory Service Broker (DSB). The tunnels can be of any technology, though it is expected that many will employ Multi-Protocol Label Switching (MPLS) or be based on optical channels. These tunnels can be both intra and inter AS in scope, in the latter case, this being achieved through operator peering. Some tunnels may offer $1+1$ protection; others may exist between peering operators through LSP stitching. However, details of the construction mechanism are considered outside the scope of this research.

Initially customers for this service are assumed to be Small and Medium Enterprises including financial institutions that wish to transport data quickly without having to incur the costs associated with a leased end-to-end infrastructure. They will have awareness of the sequence of AS domains that their data is passing through and possible alternatives, particularly if BGP reachability information is made available to them via the DSB Internet Map.

Their IT administration, which could be automated software that performs path selection based on cost and other requirements, may wish to choose a preferred path between their own site and a given destination, such as between

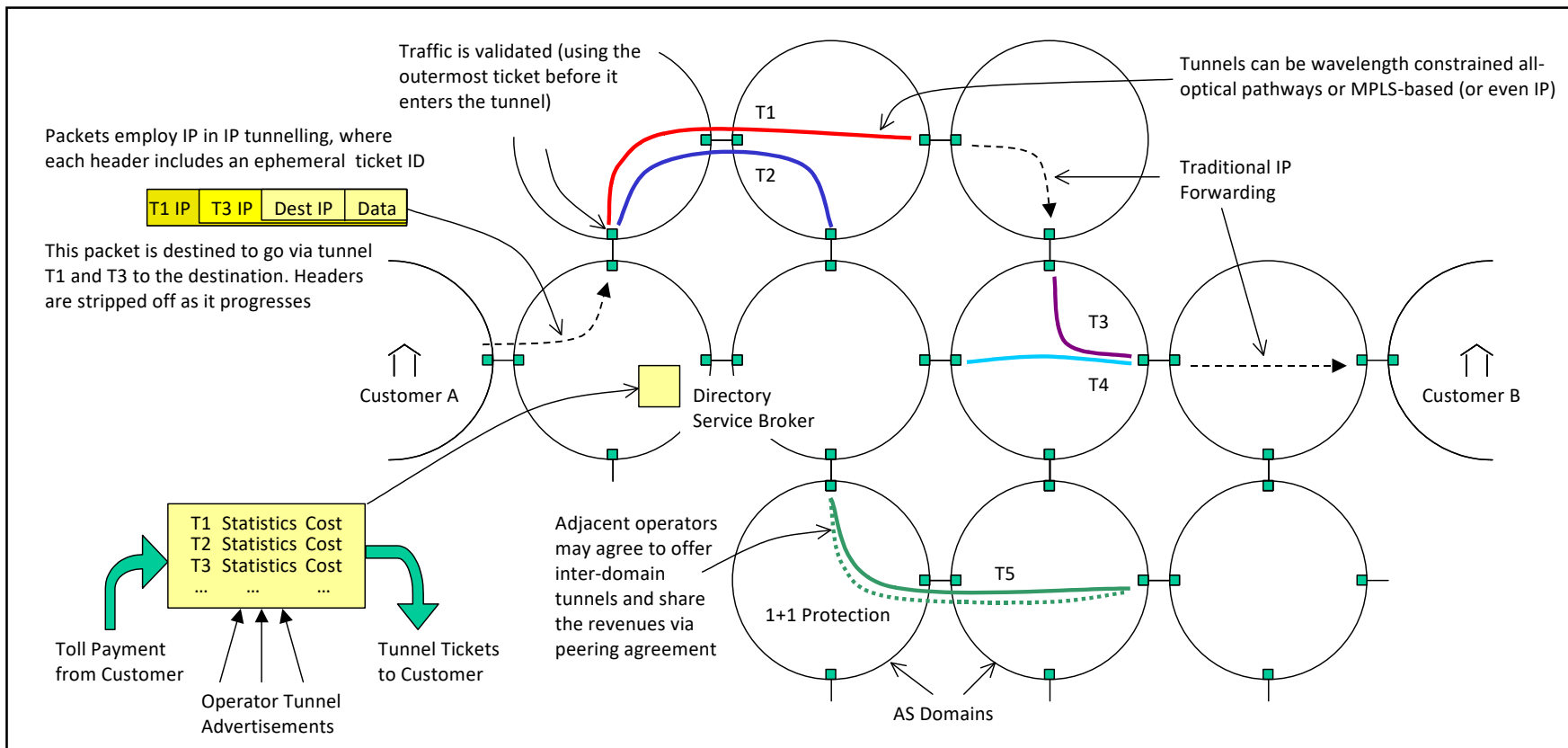

Fig. 1. . User-Selectable AS-Domain Tunnelling Framework 
Customer A and B in Fig. 1. For example, by interrogating the information in the DSB, Customer A wishes to use Tunnel T1 and T3 to hasten the delivery of data between the two sites. Having informed the DSB of this decision, for a small fee Customer A is given tickets for each of the tunnels (i.e. T1 and T3) a long with their ingress IP addresses. Tickets are ephemeral so it is unlikely that users can abuse the systemextensively.

\section{B. Network Operator Functions}

Tunnels traversing multiple domains are hampered by the unwillingness of network operators to support inter-operator signalling coupled with the control of the associated forwarding infrastructure. However, our system does not depend on any information that the network operators will not share with the Directory Service Broker (DSB) due to "trust" issues. We assume that cooperative operators will let the broker advertise their available tunnels centrally with some information such as where the tunnels are, how much the usage charge is and what performance they offer to the users. The approach does not require any sensitive information such as the mechanism by which the tunnels are established and maintained.

Our system deals with the tunnels existing between Autonomous System Border Routers (ASBRs) belonging to the same AS. There is no need to know about the internal path of the tunnels. Tunnels straddling AS domains are considered optional, as they would require a peering relationship between operators. However, mechanisms for stitching together LSPs across AS domains could technically be provided if sufficient trust existed between adjacent operators. However, what is required, is for the operators to cooperate with the broker in order to share information concerning the location of the tunnel(s) in terms of entrance and exit points, their operating performance (such as average delay, whether they are protected etc.) and the financial cost of using them.

\section{The Broker Function}

The idea of implementing a service broker has been proposed in the field of telecommunication to make an offer of the best service against a customer's request [12]. In [13], a tunnel broker system that minimizes the job of the tunnel server by assigning the broker server that handles the userrequests and returns the prime configuration to both users and tunnel servers is discussed.

We introduce a Directory Service Broker (DSB) in order to provide a centralized resource for advertising the AS tunnels to the end-users giving them the opportunity to choose to some extent their desired path across the internetwork. We assume it will have the map view of the ASes that will allow the broker to show the end users, which ASes are adjacent to each other and, in case of the cooperative ASes, information concerning their tunnels will also be available. The broker presents the location of tunnels to the users superimposed on an AS view of the Internet (or a portion of it) and the users will have the opportunity to choose whether their traffic is directed through one or more tunnels in a particular sequence. This provides a form of loose source routing. Furthermore, certain ASes may show information concerning their degree of congestion. This allows the end users to selectively choose to use a tunnel to detour traffic away from the congestion, or to provide preferential treatment across the congested AS.

We naturally as sume that the operators that are willing to cooperate will also pass some information saying whether the tunnels or their default forwarding environment are busy at a particular time and this information can be made available in the proposed broker's map view. In short, the Directory Service Broker provides an Internet Map showing the tunnel locations, their usage charge and some statistics regarding the performance they offer.

However, the broker does not tell the user how to get across the network. It provides a view of the topology, with the cost. Even there can be AS present in the network which is not cooperating with the broker. Therefore, the broker does not necessarily have complete knowledge of the network and it certainly does not know anything about what is going on in the ASes. However, it does provide AS network topology information along with a measure of how busy they are. Moreover, we have assumed that the route between the border routers (ASBRs) at the connection point between a pair of ASes is one-to-one. This is not always the case in the real Internet; rather it can be one-to-many.

The DSB also provides a single brokerage point whereby the user can request a sequence of tunnel permits (tickets) so that traffic can use a tandem arrangement of mult iple tunnels between a source and destination. The DSB is effectively the customer-facing entity where operators advertise their tunnels and the transactions that can be made.

\section{End-User Functions}

The end-user would be expected to install software in his/her network. The software would obtain the visualization part of the Internet map from the DSB. It also needs to know where the tunnels are available for the users and what is their starting and ending points. The software will get some information from the user, e.g.,

- The source and destination ASes for the data of the us er to be sent.

- Expected service of the users, where delay and other constraints will be the means to measure.

- Amount of money the user wants to pay.

Knowing the answers of the user, the software will be able to:

- Tell the least cost path using Dijkstra's Algorithm.

- Find the path with tunnels.

- Compare the constraints and the financial cost.

- Suggest the better route for the traffic.

Initially, the decision will be made depending on two factors: the benefit and the (financial) cost.

The user software will get the same visualization of the Internet map from the broker. Not necessarily all of the AS topology and ASBR topology need to be shown to the user, but it is up to the user whether it wants to have a knowledge about these. However, we are assuming that most users will be interested in getting an expected service provided with a maximum amount of financial cost it is willing to pay. In case the user wants to see the map, it is not impossible for the software to do so. Moreover, there is no concem regarding trust since no intemal details associated with this information are made available. 


\section{EVALUATION}

\section{A. Implementation}

A framework is built to investigate the benefits of using different percentages of tunnels present in a part of the Internet for sending data from one AS to another.

The topology generator tool PFP (Positive Feedback Preference) developed by Mondragon and Zhou in 2004 [14] has been used to generate regional Internet topologies which are then fed into the bespoke tool we have developed. The PFP model starts from a small random AS-graph and keeps growing where at each step, new nodes are attached to old nodes and old nodes also peer with other old nodes [14]. The probability of a node gaining a new link, which is a function of the node degree, is calculated as 0.048 [15]. The more links a node has, the more is its chance to obtain further links. The developers of PFP have explained the consequence as, "the rich not only get richer, but they get proportionately richer" $[14,15]$.

The AS topology developed from the PFP is fed into the framework developed for this research, which then produces another topology at the level of ASBRs (Autonomous Systems Border Routers), assuming there is a peering of border routers (formed by one from each of the connecting ASes) at the point where the two AS domains are connected to each other. We are aware that the route between the adjacent border routers of two connecting ASes does not necessarily have to be one-to-one; rather there can be one-toseveral connections. However, we currently confine ours elves to one-to-one ASBR peering.

Moreover, within a single $\mathrm{AS}$, the border routers are inter-connected into a full mesh, but the connections need not necessarily be direct; rather more than one internal hop may exist between a pair of border routers. Our system does not require this knowledge, nor do operators need to share this information. Therefore, the topology view of the broker is not necessarily a complete one. We can call it a "sanitized" or an "artificial" view of the Internet map. It just shows how the various ASes are inter connected at the AS level. Depending on this AS view, the ASBR topology is produced.

We then use Dijkstra's Algorithm to calculate the least cost routes for traffic to be sent from any source AS to any destination. The paths include the ASBRs that the traffic needs to traverse to reach the destination. This is the notunnel least cost path.

For now, the cost of the routes is considered using the metric of "delay" in millis econds. A data packet typically needs to go through 4 to 6 hops within a given AS while traversing across a number of ASes to reach to the destination [16]. Hence, an intra-AS tunnel having the ingress and egress points in the same AS can reduce the delay that is experienced relative to the normal no-tunnel intra-AS links. This is particularly true if the normal pathways are congested and some form of priority is given to the tunnels, be that through the use of separate optical channels or queueing priority along shared links. Fig. 2 illustrates a simp le examp le of alternate no-tunnel and tunnel paths within an AS.

In Fig. 2, the source and destination ASes are S and D and the traffic is assumed to traverse through another AS to reach the destination, which has a tunnel $\mathrm{T}$ with ingress point

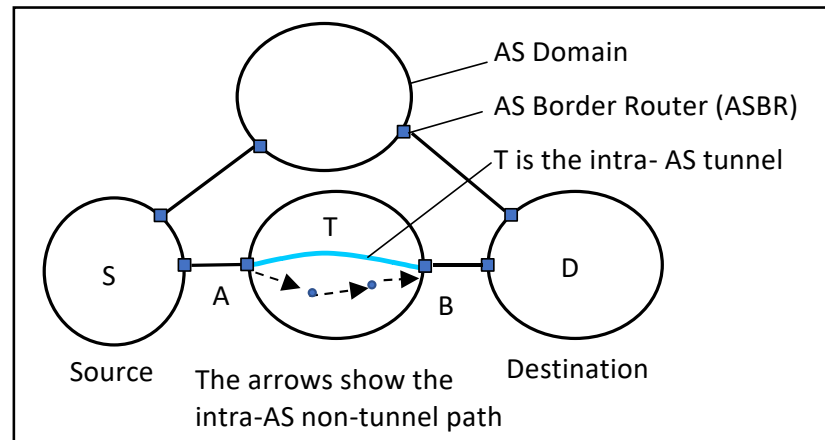

Fig. 2. Example Intra-AS paths with and without Tunnels

A and egress point B. The dotted lines represent normal intra-AS pathway including routers inside the AS.

For now, along each of the links, the associated cost is the (mean) delay in milliseconds. The four types of delay contributing to the total end-to-end delay are: transmission delay (Tx), propagation delay, processing delay and queueing delay. The propagation delay between the ASBRs $\mathrm{A}$ and $\mathrm{B}$ will be same for the no-tunnel normal path and the tunnel. [17] shows that the processing delay matters although both of processing and transmission delay are proportionately small. Hence, queueing delay is the one that typically contributes most to the delay experienced. [17] confirms that processing and queueing delays are the ones that are usually considered in terms of measurements and simulations.

The amount of delay experienced via tunnels versus notunnel intra-AS paths and the corresponding cost ratio have been chosen carefully after doing some research on Internet delay measurements $[17,18,19,20]$. Keeping the hop count in mind, our experiments have been run considering the normal intra-AS path cost as $3 \boldsymbol{x}$ milliseconds and $4 \boldsymbol{x}$ milliseconds, where the cost for using a tunnel is $\boldsymbol{x}$ milliseconds.

Our tool uses Dijkstra's Algorithm to calculate the notunnel least cost path depending on these allocated costs. After that, the tool generate a given percentage of tunnels in the produced AS topology. Taking the expected number of tunnels as user input, the tool places different percentages of tunnels in randomly chosen ASes and calculates the least cost path again considering the tunnels in and the least cost path included the tunnels if and only if the delay cost of the tunnels is less than that of the no-tunnel paths. For now, we assume an AS which is selected for hosting tunnels, has them arranged in a full mesh between the ASBRs of the AS.

\section{B. Results}

Initially, a small graph of 7 ASes is provided as an input to the PFP model in order to evolve a topology of a total of 30 ASes, where the average node degree is set as 3 and the probability of a node obtaining a new inter-AS link / adjacency is 0.04 . Using PFP, five topologies have been developed with these same characteristics that are then used to test the tooldeveloped.

Results of one of the topologies are now considered in detail. Taking the PFP-generated AS-level topology as input, the developed framework produces a topology at the ASBR level. Next Dijksta's Algorithm calculates the least cost path from every AS to all the remaining ASes. Then the presence 
of $5 \%, 10 \%, 15 \%, 20 \%, 25 \%$ and $30 \%$ tunnels are consequently added to the topology and least cost paths are again calculated for every percentage.

For all the experiments, unless otherwise stated, the ratio of the cost of a tunnel in an AS to that of a normal no-tunnel path is set as 1:3 (delays are considered in milliseconds). For now, no inter-domain tunnels have been considered and the cost of a link between the peering border routers of two adjacent ASes is set to $1 \mathrm{~ms}$.

The benefit of the tunnels present is calculated as follows:

Benefit from $A S$ " $A$ " to $A S$ " $B$ " for $x \%$ tunnels $=[$ cost from $A$ to $B$ using no tunnels minus the cost from $A$ to $B$ when $x \%$ tunnels are present] $\mathrm{ms}$

This is automatically calculated using Dijkstra's algorithm for each least cost path and then the average and standard deviation of these differences is calculated. It should be noted that in many cases there would be no cost benefit of going via one or more tunnels when they are remote from the original no-tunnel pathway.

This tunnel-placement process is repeated 10 times for a given overall AS topology and the average and standard deviation of the benefit are calculated. Fig. 3 presents a graph of the results for one topology.

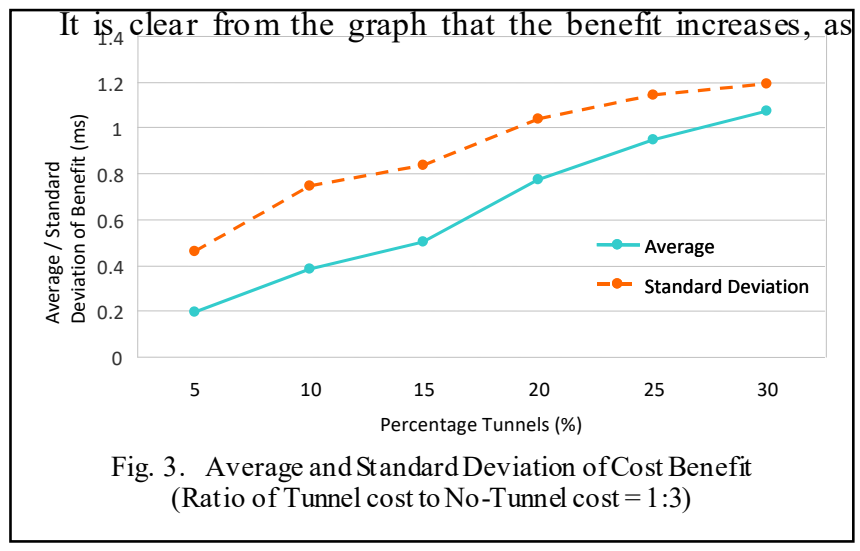

there is an increase in the percentage of tunnels present in the Internet. The average improvement is relatively small. This is not surprising, as many paths would incur a costly diversion to reach tunnel(s), particularly when they are few in number. However, the increasing standard deviation shows that between a smaller number of source-destination pairs, the cost benefit can be substantial.

\section{1) Using Different Topologies}

The same evaluation is performed for four other 30-AS topologies with the same node degree and growth characteristics. Table 2 summarises the results for those. As expected, as the proportion of tunnels increase so to does the average benefit. When the percentage of tunnels is small, the average benefit is marginal. However, from the standard deviation, we can see that some users, located close to the tunnels can still obtain a considerable benefit.
TABLE I. AVERAGE AND STANDARD DEVIATION OF THE BENEFIT OF USING TUNNELS (MS)

\begin{tabular}{|c|c|c|c|c|}
\hline $\begin{array}{c}\% \text { of } \\
\text { tunnels }\end{array}$ & Top1 & Top2 & \multicolumn{1}{|c|}{ Top3 } & \multicolumn{1}{c|}{ Top4 } \\
\hline & $\begin{array}{c}\text { Average/ } \\
\text { Std Dev }\end{array}$ & $\begin{array}{c}\text { Average/ } \\
\text { Std Dev }\end{array}$ & $\begin{array}{c}\text { Average/ } \\
\text { Std Dev }\end{array}$ & $\begin{array}{c}\text { Average/ } \\
\text { Std Dev }\end{array}$ \\
\hline $5 \%$ & $0.325057 /$ & $0.10698 /$ & $0.309425 /$ & $0.053334 /$ \\
& 0.492979 & 0.425952 & 0.601801 & 0.28789 \\
\hline $10 \%$ & $0.430345 /$ & $0.438125 /$ & $0.46006 /$ & $0.218391 /$ \\
& 0.647781 & 0.736941 & 0.790095 & 0.574282 \\
\hline $15 \%$ & $0.822418 /$ & $0.59418 /$ & $0.657011 /$ & $0.558391 /$ \\
& 0.893881 & 0.899783 & 0.953498 & 0.786062 \\
\hline $20 \%$ & $0.98318 /$ & $0.847816 /$ & $0.818391 /$ & $0.703908 /$ \\
& 1.044247 & 1.057714 & 1.049085 & 0.921514 \\
\hline $25 \%$ & $1.217595 /$ & $0.945149 /$ & $0.872184 /$ & $0.777931 /$ \\
& 1.120945 & 1.138616 & 1.092653 & 0.977332 \\
\hline $30 \%$ & $1.272562 /$ & $1.104503 /$ & $0.998161 /$ & $0.914943 /$ \\
& 1.134269 & 1.230331 & 1.173999 & 1.028713 \\
\hline
\end{tabular}

\section{2) Using a Different Cost Ratio}

The topology that was used for generating Fig. 3 is tested again for a different tunnel/ no-tunnel cost ratio of 1:4. As with the previous experiment, 10 tunnel placement runs were performed to obtain the average result. These values are

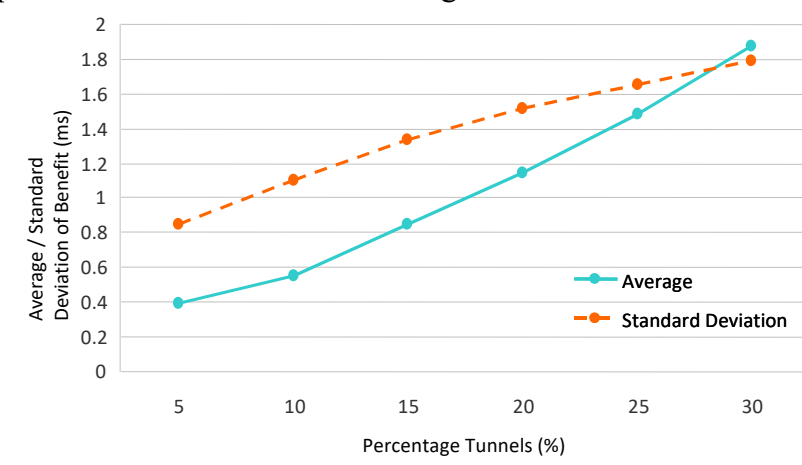

Fig. 4. Average and Standard Deviation of Cost Benefit (Ratio of Tunnel cost to No-Tunnel cost $=1: 4$ )

presented in Fig 4.

As expected, this graph shows a greater cost benefit. It is therefore worth noting that during peak hours or when specific high-demand events occur, the intra-AS queueing delay can be tens of milliseconds if not more. If tunnels bypass such "hot spots" the delay cost benefit could be orders of magnitude providing end-users considerable benefit in terms of delay reduction.

\section{3) Using a Different Average Node Degree}

Finally the PFP generator is used again to generate an AS-topology from the same initial 7-node seed graph that has been used to generate the topology used in Fig. 3 and 4. however, this time the graph evolution is altered by setting the average node degree to 4 . The tunnel / no-tunnel delay cost ratio was taken to be 1:4.

The average and standard deviation of the delay cost benefit for the presence of $5 \%, 10 \%, 15 \%, 20 \%, 25 \%$ and $30 \%$ tunnels were calculated and are shown in Fig. 5. 


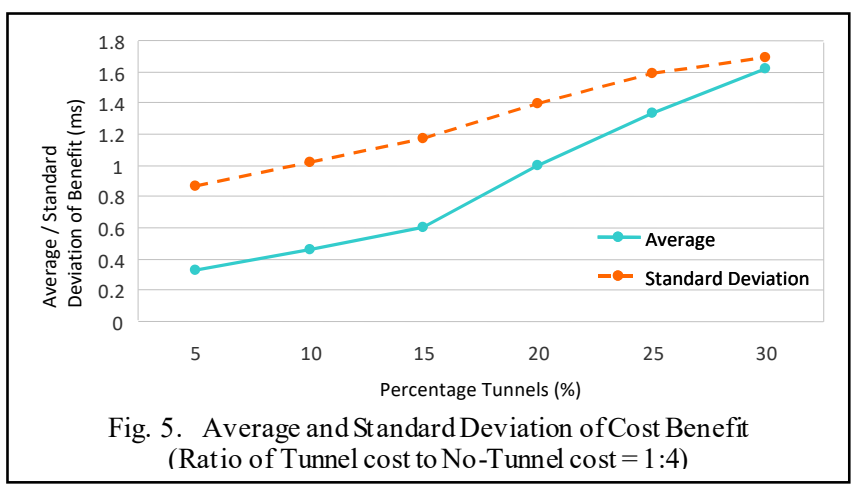

The greater inter-AS connectivity has a marginal improvement of the no-tunnel paths and thus the benefit of the tunnels is slightly reduced.

\section{DISCUSSION}

Using the developed framework we are able to examine the delay benefits that intra-AS tunnels might bring to the Internet. It shows that there is a benefit for even 5\% tunnels in the network for some users, though this is dependent on how close the tunnel alternatives are to the default traditional pathway.

To show the variation in benefit between sourcedestination tuplets we provide the standard deviation. However as one standard deviation only encompasses about $68 \%$ of a Normally distributed population, it is worth noting that for some uses the cost benefit would be appreciable. Indeed if the standard path experiences delays brought about by "hot-spot" congestion then tunnel alternatives become much more attractive. Even so, in this paper we have omitted these "extraordinary" congestion scenarios as it is selfevident that access to low delay tunnels would be attractive.

A further aspect of user-selectable tunnels not explored in detail in this paper is their ability to partially pin down a route to circumvent "less desirable" areas of the Internet.

\section{CONCLUSIONS}

This paper introduces a tunneling framework allowing cooperation between end-users and transport service providers via a brokerage mechanism. This is done in such a way that trust issues to do with the tunnel details and AS domain internal architecture are not compro mised. The paper then takes a conservative approach to the introduction of low delay-cost tunnels in an Internet region, typically compris ing about $30 \mathrm{AS}$ domains. We avoid inter-tunnels spanning ASes as this would typically require cooperation between Service Providers. Instead we focus on intra-AS tunnels that are added in a relatively low concentration. We show that although the delay-cost benefits would be marginal for many users, those users whose source-destination path is in relatively close proximity to one or more tunnels can experience a worthwhile gain. This would be more apparent as the cost differential increases. We believe that end-user selectable access to tunnels provides a suitable degree of choice whilst avoiding the issues of "net neutrality" and would allow better management of the Internet as demands on its resources continue to grow.

\section{REFERENCES}

[1] G. Schaffrath, C. Werle, P. Papadimitriou, A. Feldmann, R. Bless, A. Greenhalgh, A. Wundsam, M. Kind, O. Maennel, and L. Mathy, "Network virtualization architecture: proposal and initial prototype," Applications, Technologies, Architectures, and Protocols for Computer Communication, Proceedings of the 1st ACM workshop on Virtualized infrast ructure systems and architectures, 2009.

[2] Mohit Chamania, Marek Drogon, and Admela Jukan, "An OpenSource Path Computation Emulator: Design, Implementation, and Performance," Journal of Lightwave Technology, Vol.30, No.4, 2012.

[3] Jeffrey R. Yost, "The Origin and early history of the Computer Security Software products industry", IEEE Annals of the History of Computing, 2015.

[4] Grzegorz Rzym, Krzysztof Wajda, Krzysztof Rzym, “Analysis of PCE-based path optimization in multi-domain SDB/MPLS/BGP-LS network", 18th International Conference on Transparent Optical Networks (ICTON), 2016

[5] S. Dasgupta, et al., "Path-Computation-Element-Based Architecture for Interdomain MPLS/GMPLS Traffic Engineering: Overview and Performance," IEEE Net work Magazine, vol. 21, Issue 4, July-August 2007

[6] Adrian Farrel (2004). The Internet and Its Protocols. USA: Morgan Kaufmann. 385-489.

[7] Stefano Secci, Jean-Louis Rougier and Achille Pattavina, "On the Selection of Optimal Diverse AS-Paths for Inter-Domain IP/(G)MPLS Tunnel Provisioning", IEEE 4th International Telecommunication Networking Workshop on QoS in Multiservice IP Networks, Feb. 2008.

[8] The Guardian. (April 28, 2014). Internet service providers charging for premium access hold us all to ransom. Available: https://www.theguardian.com/technology/2014/apr/28/internet service-providers-charging-premium-access. .

[9] Wikipedia. Net neutrality. Available: https://en.wikipedia.org/wiki/Net_neutrality. Last accessed September 26, 2017

[10] Mathew Honan. (February 12, 2008). Inside Net Neutrality: Is your ISP filtering content? Available: https://www.macworld.com/article/1132075/webapps/netneutrality1 html. Last accessed August, 2018.

[11] The Guardian. (June 6, 2017). Net neutrality: Amazon among top internet firms planning day of action. Available: https://www.theguardian.com/technology/2017/jun/06/net-neutralityamazon-etsy-kickstarter-protest. Last accessed August, 2018.

[12] D. Plummer, B. Lheureux, M.Cantara, T. Bova, "Cloud Services Brokerage Is Dominated by Three Primary Roles", Gartner Research Note G00226509, 2011

[13] E. Markakis, A. Sideries, G. Alexiou, A. Bourdena, E. Pallis, G. Mastorakis and X. Macromoustakis, "A Virtual Network Functions Brokering Mechanism", International Conference on Telecommunications and Mult imedia (TEMU), IEEE, 2016

[14] S Zhou, "Characterising and Modelling the Internet Topology- the rich-club phenomenon and the PFP model," BT Technology journal, Vol 24, No 3. July 2006.

[15] R G. Clegg, Carla Gilfedder, S. Zhou, "A critical look at power law modelling of the Internet," Computer Communication, Vol 33, Issue 3, February, 2009.

[16] F. Begtasevicm P. Van Mieghem, "Measurements of the Hopcount in Internet," Submited to PAM2001, November, 2000.

[17] R. Ramaswamy, N. weng, T. Wolf, "Characterising the Network Processing Delay”, IEEE Communication Society, Globcom 2004

[18] A. Zeiroun, Chen-Nee Chuah, S. Bhattacharyya, C. Diot, "AS-Level Study of Internet Path Delay Characteristics", IEEE Communication Society, Globcom 2004.

[19] B. Choi, S. Moon, Z. Zhang, K. Papagiannaki, C. Diot, "Analysis of Point-to-Point Packet Delay In an Optimal Network," IEEE Infocom, 2004.

[20] P. Carlsson, D. Constantinescu, A. Popescu, M. Fiedler, A. Nilsson, "Delay Performance in IP Routers.” 2004 